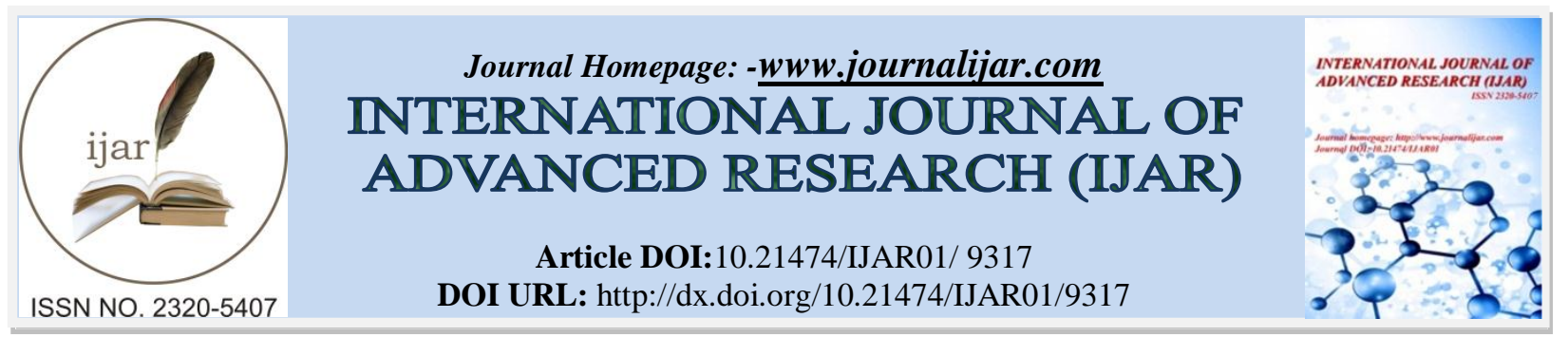

RESEARCH ARTICLE

\title{
HEREDITARY ANGIOEDEMA: AN UNUSUAL CAUSE OF VOMITING: A CASE REPORT
}

S.Ghani $^{1}$,I.Errabih $^{2}$, N. Benzzoubeir ${ }^{3}$, F.Souidine ${ }^{4}$ and L. Ouazzani ${ }^{5}$.

Hepato-Gastroenterology ans Proctology, Medicine B Department, Ibn Sina Hospital, Rabat- Morocco.

\section{Manuscript Info}

Manuscript History

Received: 02 May 2019

Final Accepted: 04 June 2019

Published: July 2019

Key words:-

Hereditary angioedema, vomiting, C1inhibitor, prophylaxis.

\begin{abstract}
Hereditary angioedema (HAE) is part of the bradykinin angioedema. It is a rare and life-threatening disease that affects about 1 in 10,000 to 50,000 people. It is a genetic disease; associated with an abnormality on the C1-inhibitor gene that leads to a deficiency of C1-inhibitor (INH), this deficiency results in uncontrolled activation of the contact system of coagulation and the classical complement pathway, resulting in excessive production of bradykinin. This disease is manifested by acute attacks of intermittent cutaneous or mucosal edema. The angioedema of the digestive tract or abdominal viscera causes intense pain, often accompanied by vomiting related to edematous intestinal obstruction, these edemas usually evolve in a few hours and persist for a few days. These crises are unpredictable and can be very serious if they affect the respiratory sphere. In this "development", after a brief review of the pathophysiology of angioedema (AE), we will detail the clinical manifestations, diagnosis and management of HAE, with a particular focus on new therapeutic agents and treatment modalities.
\end{abstract}

Copy Right, IJAR, 2019,. All rights reserved.

\section{Introduction:-}

The first clinical description of angioedema was made by Von Quincke (German physician) in 1882, and the term hereditary angioneurotic edema was coined in 1888 by Sir William Osler [1]. Hereditary angioedema (HAE) is an autosomal dominant disease, resulting from a heterozygous deficiency of $\mathrm{C} 1$-inhibitor (C1-INH), and patients with HAE have intermittent cutaneous or mucosal edema, a consequence of bad controlled local production of bradykinin. These oedemas usually evolve in a few hours and persist for a few days.

\section{Case report :}

A 39-year-old patient, followed in endocrinology since the age of 20 for hypogonadotropic hypogonadism treated by Androtardyl: 1 injection / 3 weeks, consults for recurrent abdominal pain and chronic vomiting (the patient had three episodes of vomiting monthly), with episodes of edema of the face and the hands. This symptomatology began during his childhood. His father died of laryngeal edema, two sisters and one brother also have the same symptomatology. Digestive explorations are strictly normal; including many upper endoscopy and abdominal ultrasound and CT scan, the fourth C4 components of complement was low (0.03g / dl - normal: 0.15 to 0.57$)$ as well as the concentration of $\mathrm{C} 1$-inhibitor measured by a two-fold functional test is less than $10 \%$ (normal> 50\%). The diagnosis of hereditary angioedema type I, characterized by a genetic deficiency in C1-inhibitor causing the excessive generation of bradykinin is retained. A substitution treatment with tranexamic acid (antifibrinolytic) at a dose of 1 to $1.5 \mathrm{~g} /$ day was started, with resolution of symptoms. 


\section{Discussion:-}

Angioedema associated with excess generation of bradykinin is either:hereditary, acquired or drug-induced. Hereditary angioedema (HAE) is a rare disease. The prevalence of hereditary AE due to Clinh deficiency is estimated to affect $1 / 10000$ and 1/50 000 without ethnic dominance [1]. Angioedema is caused by excessive bradykinin levels due to mutations of the SERPING1 gene. Bradykinin is a peptide released after activation of the kallikrein-kinin pathway by factor XII coagulation or Hageman's factor [2]. (Figure 1 ).

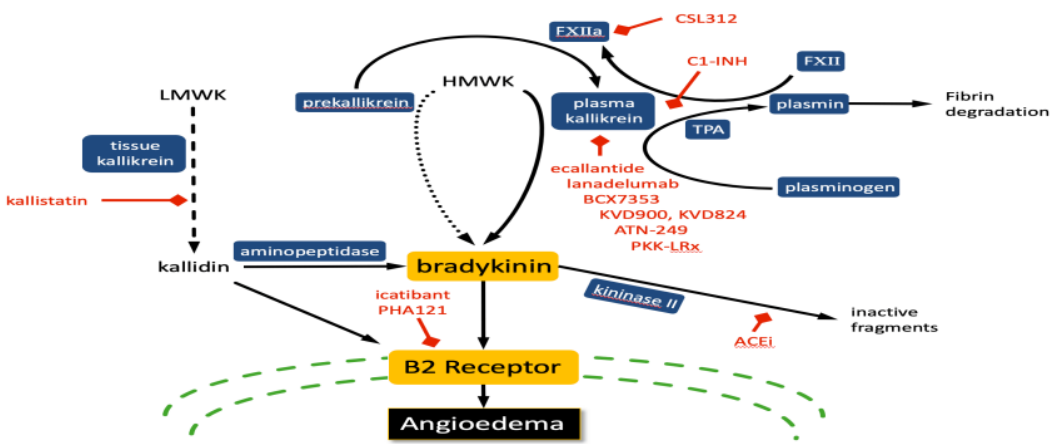

Figure 1:-Pathophysiology of angioedema [3].

Clinically [4] , Edema frequently affects the ENT area as well as the abdominal area. It is estimated that one in two patients will have laryngeal edema in their life. During the crisis, it was found that the abdominal involvement is most often present, mostly intestinal pseudo-obstruction associated to intense pain with fecal and gaz matter build up. Those pseudo-obstruction episodes lasting between 48 hours and 72 hours. The abdominal crisis is often the first symptom of the disease, and may occur several years before the first subcutaneous edema crisis[5] . The diagnosis of HAE should be confirmed through laboratory testing. Most patients with HAE have a low C1-INH concentration, which must be confirmed on two separate samples. According to consensus guidelines, the diagnosis of HAE with deficit of C1-INH should only be made when dosages of C1 -INH function are less than $50 \%$ of normal, and it should be repeated after 1-3 month. Therefore it associated with low C4 concentration level [6].

In case of severe crisis the treatment is based on the administration of Icatibant (Firazyr, $30 \mathrm{mg}$ on subcutaneous) or of C1Inh concentrate (Bérinert, $20 \mathrm{U} / \mathrm{kg}$ on intravenous), while contacting the local national referent (CREAK).

Intubation is not recommended for slight crisis wich resolve spontaneously in 2-5 days [7]. It's recommended to give Tranexamic acid (Exacyl 1 to 2 g per os every 6 hours for 24 hours) or opting for therapeutic abstention on the absence of a contraindication, this choice is left to patient.

\section{Conclusion:-}

Hereditary angioedema (HAE) is a rare disease that must be known and evoked in front of abdominal pain or unexplained vomiting with a face or hands edema, because of its potential severity and the effective therapies available. Although their clinical presentation is generally common to all types of AE. HAE have some digestive particularities not to be ignored, which should lead to the dosage of the C1-inhibitor in order to establish adequate management. Currently there are several national and international reference centers, allowing a network in all territories to facilitate diagnosis and treatments. 


\section{References:-}

1. Cicardi M, Zingale L, Zanichelli A, Pappalardo E, Cicardi B.C1 inhibitor: molecular and clinical aspects. Springer Semin Immunopathol 2005;27:286-98.

2. Walford HH, Zuraw BL. Current update on cel- lular and molecular mechanisms of hereditary angioedema. Ann Allergy Asthma Immunol 2014; 112(5):413-418.

3. C. Hermans, C. Lambert . L'angioøedème héréditaire Mécanismes, diagnostic et prise en charge. Louvain Med 2015; 134 (4): 144-152 .

4. A.Elodie (2014).les améliorations de la prise en charge des patients atteints d'angioœdèmes héréditaires. Thèse pour le Diplôme d'État de Docteur en Pharmacie. Université Angers .

5. BOUILLET L., BOCCON-GIBOD I. - L'angioœdème héréditaire en questions - Phase 5, 2010 - 40 pages .

6. A. Du-Thanh, N. Raison-Peyron, B. Guillot. Les angioedèmes à bradykinine. Annales de dermatologie et de vénéréologie (2011) 138, 328-335.

7. Longhurst H, Cicardi M. Hereditary angioedema. Lancet 2012;379:474-81. 